\title{
ON C-COMPACT ORTHOGONALLY ADDITIVE OPERATORS
}

\author{
MARAT PLIEV and MARTIN R. WEBER
}

\begin{abstract}
We consider $C$-compact orthogonally additive operators in vector lattices. After providing some examples of $C$-compact orthogonally additive operators on a vector lattice with values in a Banach space we show that the set of those operators is a projection band in the Dedekind complete vector lattice of all regular orthogonally additive operators. In the second part of the article we introduce a new class of vector lattices, called $C$-complete, and show that any laterally-to-norm continuous $C$-compact orthogonally additive operator from a $C$-complete vector lattice to a Banach space is narrow, which generalizes a result of Pliev and Popov.
\end{abstract}

\section{INTRODUCTION}

Orthogonally additive operators in vector lattices first were investigated in [12]. Later these results were extended in $[1,2,6,7,8,20,22,23])$. Recently, some connections with problems of the convex geometry were revealed [24, 25]. Orthogonally additive operators in lattice-normed spaces were studied in [3]. In this paper we continue this line of research. We analyze the notion of $C$-compact orthogonally additive operator. In the first part of the article we show that set of all $C$-compact orthogonally additive operators from a vector lattice $E$ to an order continuous Banach lattice $F$ is a projection band in the vector lattice of all regular orthogonally additive operators from $E$ to $F$ (Theorem 3.9). In the final part of the paper we introduce a new class of vector lattices which we call $C$-complete (the precise definition is given in section 4) and prove that any laterally-to-norm continuous $C$-compact orthogonally additive operator from an atomless $C$-complete vector lattice $E$ to a Banach space $X$ is narrow (Theorem 4.7). This is a generalization of the result of the article [18, Theorem 3.2].

Note that linear narrow operators in function spaces first appeared in [17]. Nowadays the theory of narrow operators is a well-studied object of functional

2010 Mathematics Subject Classification. Primary 47H30; Secondary 47H99.

Key words and phrases. C-compact operator, orthogonally additive operator, narrow operator, Urysohn operator, $C$-complete vector lattice, Banach lattice. 
analysis and is presented in many research articles $([9,11,14,15,18,23])$ and in the monograph [21].

\section{Preliminaries}

All necessary information on vector lattices one can find in [4]. In this article all vector lattices are assumed to be Archimedean.

Two elements $x, y$ of a vector lattice $E$ are called disjoint (written as $x \perp y$ ), if $|x| \wedge|y|=0$. An element $a>0$ of $E$ is an atom if $0 \leq x \leq a, 0 \leq y \leq a$ and $x \perp y$ imply that either $x=0$ or $y=0$. The equality $x=\bigsqcup_{i=1}^{n} x_{i}$ means that $x=\sum_{i=1}^{n} x_{i}$ and $x_{i} \perp x_{j}$ for all $i \neq j$. In the case of $n=2$ we write $x=x_{1} \sqcup x_{2}$. An element $y$ of a vector lattice $E$ is called a fragment (or a component) of $x \in E$, if $y \perp(x-y)$. The notation $y \sqsubseteq x$ means that $y$ is a fragment of $x$. Two fragments $x_{1}$ and $x_{2}$ of an element $x$ are said to be mutually complemented if $x=x_{1} \sqcup x_{2}$. The set of all fragments of an element $x \in E$ is denoted by $\mathcal{F}_{x}$.

Definition 2.1. Let be $E$ a vector lattice and $X$ a real vector space. An operator $T: E \rightarrow X$ is said to be orthogonally additive if $T(x+y)=T x+T y$ for any disjoint elements $x, y \in E$.

It is not hard to check that $T(0)=0$. The set of all orthogonally additive operators from $E$ to $X$ is a real vector space with respect to the natural linear operations.

Definition 2.2. Let $E$ and $F$ be vector lattices. An orthogonally additive (in general, nonlinear) operator $T: E \rightarrow F$ is said to be:

- positive if $T x \geq 0$ for all $x \in E$,

- regular, if $T=S_{1}-S_{2}$ for two positive, orthogonally additive operators $S_{1}$ and $S_{2}$ from $E$ to $F$.

The sets of all positive, regular orthogonally additive operators from $E$ to $F$ are denoted by $\mathcal{O} \mathcal{A}_{+}(E, F), \mathcal{O} \mathcal{A}_{r}(E, F)$, respectively, where the order in $\mathcal{O} \mathcal{A}_{r}(E, F)$ is introduced as follows: $S \leq T$ whenever $(T-S) \geq 0$. Then $\mathcal{O} \mathcal{A}_{r}(E, F)$ becomes an ordered vector space.

For a Dedekind complete vector lattice $F$ we have the following property of $\mathcal{O} \mathcal{A}_{r}(E, F)$.

Proposition 2.3 ([19, Theorem 3.6]). Let $E$ and $F$ be a vector lattices, and assume that $F$ is Dedekind complete.Then $\mathcal{O A}_{r}(E, F)$ is a Dedekind complete 
vector lattice. Moreover, for every $S, T \in \mathcal{O A}_{r}(E, F)$ and every $x \in E$ the following formulas ${ }^{1}$ hold

(1) $(T \vee S)(x)=\sup \{T y+S z: x=y \sqcup z\}$;

(2) $(T \wedge S)(x)=\inf \{T y+S z: x=y \sqcup z\}$;

(3) $T^{+}(x)=\sup \{T y: y \sqsubseteq x\}$;

(4) $T^{-}(x)=-\inf \{T y: y \sqsubseteq x\}$;

(5) $|T x| \leq|T|(x)$.

\section{The Projection band of $C$-COMpaCt orthogonally additive OPERATORS}

In this section we show that the set of all $C$-compact regular orthogonally additive operators from a vector lattice $E$ to a Banach lattice $F$ with order continuous norm is a band in the vector lattice of all orthogonally additive regular operators from $E$ to $F$.

Consider some examples.

Example 3.1. Assume that $(A, \Xi, \mu)$ and $(B, \Sigma, \nu)$ are $\sigma$-finite measure spaces. We say that a map $K: A \times B \times \mathbb{R} \rightarrow \mathbb{R}$ is a Carathéodory function if there hold the conditions:

(1) $K(\cdot, \cdot, r)$ is $\mu \times \nu$-measurable for all $r \in \mathbb{R}$;

(2) $K(s, t, \cdot)$ is continuous on $\mathbb{R}$ for $\mu \times \nu$-almost all $(s, t) \in A \times B$.

If additionally there holds $K(s, t, \cdot)=0$ for $\mu \times \nu$-almost all $(s, t) \in A \times B$ then with the function $K$ a nonlinear integral operator: $T: D o m(K) \rightarrow L_{0}(\mu)$

$$
(T f)(s):=\int_{B} K(s, t, f(t)) d \nu(t) \quad \text { for } \mu \text {-almost all } s \in A
$$

is associated, where

$$
\operatorname{Dom}(K):=\left\{f \in L_{0}(\nu): \int_{B}|K(s, t, f(t))| d \nu(t) \in L_{0}(\mu)\right\} .
$$

Assume that $E$ is a Banach function space in $L_{0}(\nu)$ and $E \subseteq \operatorname{Dom}(K)$. Then $T$ defines an orthogonally additive integral operator acting from $E$ to $L_{0}(\mu)$. The operator $T$ is called Urysohn (integral) operator and, the function $K$ is called the kernel of this operator. If $L_{0}(\mu)$ is replaced by $\mathbb{R}$ we say that $T$ is an Urysohn integral functional.

\footnotetext{
${ }^{1}$ In the literature these formulas are known as the Riesz-Kantorovich formulas.
} 
Example 3.2. Assume that $(\Omega, \Sigma, \mu)$ is a measure space. Then the functional $\mathcal{N}: L_{1}(\mu) \rightarrow \mathbb{R}$ defined by

$$
\mathcal{N}(f)=\|f\|_{L_{1}(\mu)}:=\int_{\Omega}|f(t)| d \mu, \quad f \in L_{1}(\mu)
$$

is positive and orthogonally additive.

Let $E$ be a vector lattice. A linear operator $S: E \rightarrow E$ is called band preserving if $S x \in\{x\}^{\perp \perp}$ for all $x \in E$. Clearly, every band preserving operators preserves disjointness.

Example 3.3. Let $(\Omega, \Sigma, \mu)$ be a measure space and $S: L_{0}(\mu) \rightarrow L_{0}(\mu)$ be a band preserving linear operator. Consider the new operator $T: L_{0}(\mu) \rightarrow L_{0}(\mu)$ defined by

$$
T f=f S(f), \quad f \in L_{0}(\mu) .
$$

Observe that $T$ can be treated as the multiplication operator by the "variable" function. It is not hard to verify that $T$ is an orthogonally additive operator. Indeed, due to $S f \in\{f\}^{\perp \perp}$, for every disjoint $f_{1}, f_{2} \in L_{0}(\mu)$ we have

$$
\begin{array}{r}
T\left(f_{1}+f_{2}\right)=\left(f_{1}+f_{2}\right) S\left(f_{1}+f_{2}\right)= \\
f_{1} S\left(f_{1}+f_{2}\right)+f_{2} S\left(f_{1}+f_{2}\right)= \\
f_{1} S f_{1}+f_{2} S f_{2}=T f_{1}+T f_{2} .
\end{array}
$$

Definition 3.4. Let $(\Omega, \Sigma, \mu)$ be a $\sigma$-finite measure space. We say that a function $N: \Omega \times \mathbb{R} \rightarrow \mathbb{R}$ belongs to the class $\mathfrak{S}$ (or $N$ is a $\mathfrak{S}$-function) if the following conditions hold:

(1) $N(t, 0)=0$ for $\mu$-almost all $t \in \Omega$;

(2) $N(\cdot, g(\cdot))$ is $\mu$-measurable for any $g \in L_{0}(\mu)$.

If a function $N$ satisfies only the condition (2), then it is called a superpositionally measurable function or sup-measurable function.

Example 3.5. Let $N: \Omega \times \mathbb{R} \rightarrow \mathbb{R}$ be a $\mathfrak{S}$-function. Then with $N$ there is associated an orthogonally additive operator $T_{N}: L_{0}(\mu) \rightarrow L_{0}(\mu)$ defined by

$$
T_{N}(g)(t)=N(t, g(t)) \text { for } \mu \text {-almost all } t \in \Omega \text { and } g \in L_{0}(\mu) .
$$

This class of operators (sometimes called nonlinear superposition operators or Nemytskii operators) is widely represented in the literature (see e.g. [5]).

Definition 3.6. Let be $E$ be a vector lattice and $Y$ a normed space. An orthogonally additive operator $T: E \rightarrow Y$ is called 
- AM-compact provided $T$ maps order bounded subsets of $E$ into relatively compact subsets in $Y$,

- $C$-compact, if $T\left(\mathcal{F}_{x}\right)$ is a relatively compact in $Y$ for any $x \in E$.

For a Banach lattice $F$ by $\mathcal{C O} \mathcal{A}_{r}(E, F)$ is denoted the space of all $C$-compact regular orthogonally additive operators from $E$ to $F$.

Example 3.7. We note that $\mathcal{O} \mathcal{A}_{r}(\mathbb{R}, \mathbb{R})$ is exactly the set of all real-valued functions such that $f(0)=0$. Define an orthogonally additive operator $T: \mathbb{R} \rightarrow \mathbb{R}$ by

$$
T x= \begin{cases}\frac{1}{x^{2}}, & \text { if } x \neq 0 \\ 0, & \text { if } x=0 .\end{cases}
$$

Since any element $x \in \mathbb{R}$ is an atom one has $\mathcal{F}_{x}=\{0, x\}$ for any $x \in \mathbb{R}$. It follows that $T$ is a $C$-compact operator. On the other hand $T([0,1])$ is an unbounded set in $\mathbb{R}$ and therefore $T$ is not AM-compact.

Proposition 3.8. Let $(B, \Sigma, \nu)$ be $\sigma$-finite measure spaces $E$ be a Banach function space in $L_{0}(\nu)$ and $T: E \rightarrow \mathbb{R}$ be the Urysohn integral functional defined by

$$
T f=\int_{B} K(t, f(t)) d \nu(t), \quad f \in E
$$

with the kernel $K$. Then $T$ is $C$-compact.

Proof. Take $f \in E$. We note that $\mathcal{F}_{f}$ coincides with the set $\left\{f \mathbf{1}_{D}: D \in \Sigma\right\}$. Now for every $D \in \Sigma$ we write

$$
\begin{array}{r}
T f \mathbf{1}_{D}=\int_{B} K\left(t, f \mathbf{1}_{D}(t)\right) d \nu(t)=\int_{D} K(t, f(t)) d \nu(t) \leq \\
\int_{B}|K(t, f(t))| d \nu(t)=M .
\end{array}
$$

Hence the set $T\left(\mathcal{F}_{f}\right)$ is order bounded in $\mathbb{R}$ and therefore the operator $T$ is $C$-compact.

We mention that a $C$-compact order bounded orthogonally additive operator $T: E \rightarrow F$ from a Banach lattices $E$ to a $\sigma$-Dedekind complete Banach lattice $F$ is $A M$-compact if, in addition, $T$ is uniformly continuous on order bounded subsets of $E$ [13, Theorem 3.4].

The norm in a normed vector lattice is order continuous if $x_{\alpha} \downarrow 0$ implies $\left\|x_{\alpha}\right\| \downarrow 0$. We point out that a Banach lattice with order continuous norm is Dedekind complete (see [4, Theorem 12.9]).

Now we are ready to present the first main result of the article. 
Theorem 3.9. Let be $E$ a vector lattice and $F$ a Banach lattice with order continuous norm. Then the set of all $C$-compact regular orthogonally additive operators from $E$ to $F$ is a projection band in $\mathcal{O} \mathcal{A}_{r}(E, F)$.

In order to prove Theorem 3.9 we need some auxiliary propositions.

Proposition 3.10. [16, Proposition 3.9] Let $E$ be a vector lattice and $x, y \in E$. Then $x \sqsubseteq y$ if and only if $x^{+} \sqsubseteq y^{+}$and $x^{-} \sqsubseteq y^{-}$.

Proposition 3.11. Let $E$ be a vector lattice and $\bigsqcup_{i=1}^{n} x_{i}=\bigsqcup_{k=1}^{m} y_{k}$ for some $\left(x_{i}\right)_{i=1}^{n}$ and $\left(y_{k}\right)_{k=1}^{m} \subset E$. Then there exist a family of pairwise disjoint elements $\left(z_{i k}\right) \subset$ $E$, where $i \in\{1, \ldots, n\}$ and $k \in\{1, \ldots, m\}$ such that

(i) $x_{i}=\bigsqcup_{k=1}^{m} z_{i k}$ for any $i \in\{1, \ldots, n\}$;

(ii) $y_{k}=\bigsqcup_{i=1}^{n} z_{i k}$ for any $k \in\{1, \ldots, m\}$;

(iii) $\bigsqcup_{i=1}^{n} \bigsqcup_{k=1}^{m} z_{i k}=\bigsqcup_{i=1}^{n} x_{i}=\bigsqcup_{k=1}^{m} y_{k}$.

Proof. Using Proposition 3.10, and applying induction arguments we have

$$
\begin{aligned}
& \bigsqcup_{i=1}^{n} x_{i}^{+}=\left(\bigsqcup_{i=1}^{n} x_{i}\right)^{+}=\left(\bigsqcup_{k=1}^{m} y_{k}\right)^{+}=\bigsqcup_{k=1}^{m} y_{k}^{+} \\
& \bigsqcup_{i=1}^{n} x_{i}^{-}=\left(\bigsqcup_{i=1}^{n} x_{i}\right)^{-}=\left(\bigsqcup_{k=1}^{m} y_{k}\right)^{-}=\bigsqcup_{k=1}^{m} y_{k}^{-} .
\end{aligned}
$$

Now, by the Riesz Decomposition property there exist

$$
z_{i k}^{+}, z_{i k}^{-} \in E_{+}, \quad i \in\{1, \ldots, n\} \text { and } k \in\{1, \ldots, m\}
$$

such that

$$
\begin{aligned}
& x_{i}^{+}=\bigsqcup_{k=1}^{m} z_{i k}^{+}, \quad x_{i}^{-}=\bigsqcup_{k=1}^{m} z_{i k}^{-}, \quad i \in\{1, \ldots, n\}, \\
& y_{k}^{+}=\bigsqcup_{i=1}^{n} z_{i k}^{+}, \quad y_{k}^{-}=\bigsqcup_{i=1}^{n} z_{i k}^{-}, \quad k \in\{1, \ldots, m\} .
\end{aligned}
$$

Set $z_{i k}=z_{i k}^{+}-z_{i k}^{-}, \quad i \in\{1, \ldots, n\}$ and $k \in\{1, \ldots, m\}$. Hence

$$
\bigsqcup_{i=1}^{n} \bigsqcup_{k=1}^{m} z_{i k}=\bigsqcup_{i=1}^{n} x_{i}=\bigsqcup_{k=1}^{m} y_{k}
$$

and the proof is completed. 
Lemma 3.12. Let $E$ and $F$ be vector lattices with $F$ Dedekind complete, $S, T \in$ $\mathcal{O} \mathcal{A}_{r}(E, F)$ and $x \in E$. Then the following equalities hold:

$$
\begin{aligned}
& \text { (1) }(T \vee S) x=\sup \left\{\sum_{i=1}^{n} T x_{i} \vee S x_{i}: x=\bigsqcup_{i=1}^{n} x_{i}, n \in \mathbb{N}\right\} ; \\
& \text { (2) }(T \wedge S) x=\inf \left\{\sum_{i=1}^{n} T x_{i} \wedge S x_{i}: x=\bigsqcup_{i=1}^{n} x_{i}, n \in \mathbb{N}\right\} ; \\
& \text { (3) }|T| x=\sup \left\{\sum_{i=1}^{n}\left|T x_{i}\right|: x=\bigsqcup_{i=1}^{n} x_{i}, n \in \mathbb{N}\right\} .
\end{aligned}
$$

Proof. Since $x \wedge y=-(-x) \vee(-y)$ and $|x|=x \vee(-x)$ for proving the lemma it is sufficient to establish only the equation (1). Put

$$
\mathfrak{A}(x):=\left\{\sum_{i=1}^{n} T x_{i} \vee S x_{i}: x=\bigsqcup_{i=1}^{n} x_{i}, n \in \mathbb{N}\right\} .
$$

We show that $\mathfrak{A}(x)$ is an upward directed set. Indeed, take two disjoint decompositions $x=\bigsqcup_{i=1}^{n} x_{i}$ and $x=\bigsqcup_{k=1}^{m} y_{k}$ of $x$. By Proposition 3.11 there exists a disjoint decomposition $x=\bigsqcup_{i=1}^{n} \bigsqcup_{k=1}^{m} z_{i k}$ such that

$$
\begin{gathered}
x_{i}=\bigsqcup_{k=1}^{m} z_{i k}, \quad i \in\{1, \ldots, n\}, \\
y_{k}=\bigsqcup_{i=1}^{n} z_{i k}, \quad k \in\{1, \ldots, m\} .
\end{gathered}
$$

We observe that

$$
S x_{i} \vee T x_{i} \leq \sum_{k=1}^{m} T z_{i k} \vee S z_{i k}
$$

for any $i \in\{1, \ldots, n\}$. Indeed

$$
\begin{gathered}
S x_{i}=S\left(\bigsqcup_{k=1}^{m} z_{i k}\right)=\sum_{k=1}^{m} S z_{i k} \leq \sum_{k=1}^{m} T z_{i k} \vee S z_{i k}, \\
T x_{i}=T\left(\bigsqcup_{k=1}^{m} z_{i k}\right)=\sum_{k=1}^{m} T z_{i k} \leq \sum_{k=1}^{m} T z_{i k} \vee S z_{i k} \Longrightarrow \\
S x_{i} \vee T x_{i} \leq \sum_{k=1}^{m} T z_{i k} \vee S z_{i k} .
\end{gathered}
$$

Similar arguments show that

$$
S y_{k} \vee T y_{k} \leq \sum_{i=1}^{n} T z_{i k} \vee S z_{i k}
$$


for any $k \in\{1, \ldots, m\}$. Now we may write

$$
\sum_{i=1}^{n} T x_{i} \vee S x_{i} \leq \sum_{i=1}^{n} \sum_{k=1}^{m} T z_{i k} \vee S z_{i k} \quad \text { and } \quad \sum_{k=1}^{m} T y_{k} \vee S y_{k} \leq \sum_{i=1}^{n} \sum_{k=1}^{m} T z_{i k} \vee S z_{i k}
$$

and deduce that $\mathfrak{A}(x)$ is an upward directed subset of $F$. By Proposition 2.3 $\mathcal{O} \mathcal{A}_{r}(E, F)$ is a Dedekind complete vector lattice and

$$
(T \vee S) x=\sup \{T y+S z: x=y \sqcup z\} .
$$

Clearly $T x \vee S x \leq(T \vee S) x$ for any $x \in E$. Thus for any decomposition $x=$ $\bigsqcup_{i=1}^{n} x_{i}, n \in \mathbb{N}$ we have that

$$
\sum_{i=1}^{n} T x_{i} \vee S x_{i} \leq \sum_{i=1}^{n}(T \vee S) x_{i}=(T \vee S) x
$$

and therefore the set $\mathfrak{A}(x)$ is upper order bounded. Put $f_{x}:=\sup \mathfrak{A}(x)$. Then $f_{x} \leq(T \vee S) x$. On the other hand for any disjoint decomposition $x=y \sqcup z$ we have

$$
\begin{gathered}
T y+S z \leq \sup \{T y \vee S y+T z \vee S z: x=y \sqcup z\} \leq \\
\sup \left\{\sum_{i=1}^{n} T x_{i} \vee S x_{i}: x=\bigsqcup_{i=1}^{n} x_{i}, n \in \mathbb{N}\right\}=f_{x} .
\end{gathered}
$$

Passing to the supremum in the last inequality over all disjoint decompositions $x=y \sqcup z$ of $x$, due to formula (1) of Proposition 2.3, we deduce $(T \vee S) x \leq f_{x}$. It follows $(T \vee S) x=f_{x}$ which completes the proof.

Below we shall use the following elementary observation. Since

$$
\left\{\sum_{i=1}^{n} S x_{i} \vee T x_{i}: x=\bigsqcup_{i=1}^{n} x_{i}, n \in \mathbb{N}\right\}
$$

is an upward downward directed subset of $F$ the set

$$
\left\{\sum_{i=1}^{n} S x_{i} \wedge T x_{i}: x=\bigsqcup_{i=1}^{n} x_{i}, n \in \mathbb{N}\right\}
$$

is downward directed.

Lemma 3.13. Let be $E$ a vector lattice, $F$ a Banach lattice with order continuous norm and $T \in \mathcal{C O} \mathcal{A}_{r}(E, F)$. Then $\mathcal{F}_{T} \subset \mathcal{C O} \mathcal{A}_{r}(E, F)$.

Proof. We recall that by definition $S \in \mathcal{F}_{T}$ if $|S| \wedge|T-S|=0$. We show that $S\left(\mathcal{F}_{x}\right)$ is a relatively compact $\operatorname{set}^{2}$ in $F$ for any $S \in \mathcal{F}_{T}$ and $x \in E$. Indeed, fix

\footnotetext{
2 Due to the norm-completeness of $F$ this is equivalent to its totally boundedness.
} 
$x \in E$ and $\varepsilon>0$. Since $\left\{\sum_{i=1}^{n}|S| x_{i} \wedge|T-S| x_{i}: x=\bigsqcup_{i=1}^{n} x_{i}, n \in \mathbb{N}\right\}$ is a downward directed subset of $F$ by Lemma 3.12 and the order continuity of the norm in the Banach lattice $F$ there exists a disjoint decomposition $x=\bigsqcup_{i=1}^{n} x_{i}$ of $x$ such that

$$
\left\|\sum_{i=1}^{n}|S| x_{i} \wedge|T-S| x_{i}\right\|<\frac{\varepsilon}{4}
$$

Since $T\left(\mathcal{F}_{x_{i}}\right)$ is a relatively compact set for any $i \in\{1, \ldots, n\}$ there exists a finite subset $D_{i} \subset \mathcal{F}_{x_{i}}$ such that for any $w \in \mathcal{F}_{x_{i}}$ there is $u \in D_{i}$ satisfying

$$
\|T w-T u\|<\frac{\varepsilon}{2 n} .
$$

Moreover, by Proposition 3.11 for any $y \in \mathcal{F}_{x}$ there exists a disjoint decomposition $y=\bigsqcup_{i=1}^{n} y_{i}$, where $y_{i} \sqsubseteq x_{i}$, i.e. $y_{i} \in \mathcal{F}_{x_{i}}$. Hence, for some $u_{i} \in D_{i}$ the following inequality

$$
\left\|T y_{i}-T u_{i}\right\|<\frac{\varepsilon}{2 n}
$$

holds. We remark that for any positive orthogonally additive operator $G: E \rightarrow F$ one has ${ }^{3} G w \leq G x_{i}$ for all $w \in \mathcal{F}_{x_{i}}$. Now, by taking into account the inequality $|x| \leq|x+y|+|x| \wedge|y|$, which holds in any vector lattice ${ }^{4}$ we have

$$
\begin{aligned}
& \left|S y_{i}-S u_{i}\right| \leq \\
& \left|S y_{i}-S u_{i}+(T-S) y_{i}-(T-S) u_{i}\right|+\left|S y_{i}-S u_{i}\right| \wedge\left|(T-S) y_{i}-(T-S) u_{i}\right| \leq \\
& \left|T y_{i}-T u_{i}\right|+\left(\left|S y_{i}\right|+\left|S u_{i}\right|\right) \wedge\left(\left|(T-S) y_{i}\right|+\left|(T-S) u_{i}\right|\right) \leq \\
& \left|T y_{i}-T u_{i}\right|+\left(|S| y_{i}+|S| u_{i}\right) \wedge\left(|T-S| y_{i}+|T-S| u_{i}\right) \leq \\
& \left|T y_{i}-T u_{i}\right|+\left(|S| x_{i}+|S| x_{i}\right) \wedge\left(|T-S| x_{i}+|T-S| x_{i}\right)= \\
& \left|T y_{i}-T u_{i}\right|+2\left(|S| x_{i} \wedge|T-S| x_{i}\right) .
\end{aligned}
$$

Then we may write

$$
\sum_{i=1}^{n}\left|S y_{i}-S u_{i}\right| \leq \sum_{i=1}^{n}\left|T y_{i}-T u_{i}\right|+2 \sum_{i=1}^{n}\left(|S| x_{i} \wedge|T-S| x_{i}\right)
$$

\footnotetext{
${ }^{3}$ This immediately follows from $|y| \wedge|x-y|=0$, the positivity of $G$ and $G(x)=G(y)+$ $G(x-y) \geq G(y)$.

${ }^{4}$ Indeed, since (see [26], Theorems 5.1 and 5.5) $|x+y| \geq|| x|-| y|| \geq(|x|-|y|)^{+}$we have $|x|-|x| \wedge|y|=|x|+(-|x|) \vee(-|y|)=0 \vee(|x|-|y|)=(|x|-|y|)^{+} \leq|x+y|$.
} 
Put $D:=\left\{u=\bigsqcup_{i=1}^{n} u_{i}: u_{i} \in D_{i}\right\}$. Clearly $D$ is a finite subset of $\mathcal{F}_{x}$. Thus for any $y \in \mathcal{F}_{x}$ and $u \in D$ we have

$$
\begin{aligned}
& \|S y-S u\|=\left\|\left|S\left(\bigsqcup_{i=1}^{n} y_{i}\right)-S\left(\bigsqcup_{i=1}^{n} u_{i}\right)\right|\right\|=\left\|\left|\sum_{i=1}^{n} S y_{i}-S u_{i}\right|\right\| \leq \\
& \left\|\sum_{i=1}^{n}\left|S y_{i}-S u_{i}\right|\right\| \leq\left\|\sum_{i=1}^{n}\left|T y_{i}-T u_{i}\right|\right\|+2\left\|\sum_{i=1}^{n}\left(|S| x_{i} \wedge|T-S| x_{i}\right)\right\| \leq \\
& \sum_{i=1}^{n}\left\|T y_{i}-T u_{i}\right\|+\frac{\varepsilon}{2} \leq \varepsilon .
\end{aligned}
$$

Hence $\{S u: u \in D\}$ is a finite $\varepsilon$-net for $S\left(\mathcal{F}_{x}\right)$, and therefore the proof is completed.

Let $V$ be a vector lattice and $v \in V_{+}$. The order ideal generated by $v$ is denoted by $I_{v}$, i.e. $I_{v}=\{x \in V: \exists \alpha>0$ such that $|x| \leq \alpha|v|\}$. A $v$-step function is any vector $s \in V$ for which there exist pairwise disjoint fragments $v_{1}, \ldots, v_{n}$ of $v$ with $v=\bigsqcup_{i=1} v_{i}^{n}$ and real numbers $\lambda_{1}, \ldots, \lambda_{n}$ satisfying $s=\sum_{i=1}^{n} \lambda_{i} v_{i}$. The next proposition is known as the Freudenthal Spectral Theorem ([4], Theorem 2.8).

Proposition 3.14. Let $V$ be a vector lattice with the principal projection property and let $v \in V_{+}$. Then for every $u \in I_{v}$ there exists a sequence $\left(s_{n}\right)_{n \in \mathbb{N}}$ of $v$-step functions satisfying $0 \leq u-s_{n} \leq \frac{1}{n} v$ for each $n$ and $s_{n} \uparrow u$. Moreover, $0 \leq s_{n}$ if $0 \leq u$.

Now we ready to prove the first main result.

Proof of Theorem 3.9. We prove some properties of $\mathcal{C O} \mathcal{A}_{r}(E, F)$ :

a) Clearly, $\mathcal{C O} \mathcal{A}_{r}(E, F)$ is a vector subspace of $\mathcal{O} \mathcal{A}_{r}(E, F)$.

b) We show that $\mathcal{C O} \mathcal{A}_{r}(E, F)$ is even a vector sublattice of $\mathcal{O} \mathcal{A}_{r}(E, F)$. Take $S, T \in \mathcal{C O} \mathcal{A}_{r}(E, F)$. Then $T-S \in \mathcal{C O} \mathcal{A}_{r}(E, F)$. By Lemma $3.13 \mathcal{F}_{T} \subset$ $\mathcal{C O} \mathcal{A}_{r}(E, F)$ and therefore $T^{+} \in \mathcal{C O} \mathcal{A}_{r}(E, F)$. Therefore due to the equalities

$$
S+(T-S)^{+}=S+(T-S) \vee 0=T \vee S, \quad S \wedge T=-(-S) \vee(-T)
$$

which are valid in $\mathcal{O A}_{r}(E, F)$ we obtain that $\mathcal{C O} \mathcal{A}_{r}(E, F)$ is a sublattice of $\mathcal{O} \mathcal{A}_{r}(E, F)$.

c) Now we show that $T \in \mathcal{C O} \mathcal{A}_{r}(E, F)$ if $0 \leq T_{\lambda} \uparrow T$ in $\mathcal{O} \mathcal{A}_{r}(E, F)$ and any $T_{\lambda} \in \mathcal{C O} \mathcal{A}_{r}(E, F)$. Indeed, take $x \in E$ and $\varepsilon>0$. Since the Banach lattice $F$ is order continuous it follows from $T_{\lambda} x \uparrow T x$ that $\left\|T x-T_{\lambda_{0}} x\right\|<\frac{\varepsilon}{4}$ for some $\lambda_{0}$. 
Then actually $\left\|T y-T_{\lambda_{0}} y\right\|<\frac{\varepsilon}{4}$ for any $y \in \mathcal{F}_{x}$. Indeed, consider $x=y \sqcup z$ for some $z \in E$. Then

$$
0 \leq T x-T_{\lambda_{0}} x=T(y \sqcup z)-T_{\lambda_{0}}(y \sqcup z)=T y-T_{\lambda_{0}} y+T z-T_{\lambda_{0}} z \geq T y-T_{\lambda_{0}} y
$$

implies $\left\|T y-T_{\lambda_{0}} y\right\| \leq\left\|T x-T_{\lambda_{0}} x\right\|$. Since $T_{\lambda_{0}} \in \mathcal{C O} \mathcal{A}_{r}(E, F)$ there exists a finite subset $D$ of $\mathcal{F}_{x}$ with the property that for any $y \in \mathcal{F}_{x}$ there exists $u \in D$ satisfying

$$
\left\|T_{\lambda_{0}} u-T_{\lambda_{0}} y\right\|<\frac{\varepsilon}{2}
$$

So we obtain

$$
\begin{array}{r}
\|T u-T y\| \leq\left\|T u-T_{\lambda_{0}} u+T_{\lambda_{0}} u-T y+T_{\lambda_{0}} y-T_{\lambda_{0}} y\right\| \leq \\
\left\|T u-T_{\lambda_{0}} u\right\|+\left\|T y-T_{\lambda_{0}} y\right\|+\left\|T_{\lambda_{0}} u-T_{\lambda_{0}} y\right\|<\varepsilon,
\end{array}
$$

what establishes the relative compactness of $T\left(\mathcal{F}_{T}\right)$ in $F$.

d) Finally we prove that $\mathcal{C O} \mathcal{A}_{r}(E, F)$ is an order ideal in $\mathcal{O A}_{r}(E, F)$. Let $0 \leq R \leq T$, where $R \in \mathcal{O A}_{r}(E, F)$ and $T \in \mathcal{C O} \mathcal{A}_{r}(E, F)$. Then $R \in I_{T}$ and by Proposition 3.14 there $^{5}$ exists a sequence $\left(S_{n}\right)_{n \in \mathbb{N}}$ in $\mathcal{O A}_{r}(E, F)$ of $T$ step-functions with $0 \leq S_{n} \uparrow R$. Taking into account that ${ }^{6} S_{n} \in \mathcal{C O} \mathcal{A}_{r}(E, F)$, $n \in \mathbb{N}$ and what has been established in c) we deduce that $R \in \mathcal{C O} \mathcal{A}_{r}(E, F)$. So, $\mathcal{C O} \mathcal{A}_{r}(E, F)$ is a band in $\mathcal{O} \mathcal{A}_{r}(E, F)$.

e) Due to the Dedekind completeness of $\mathcal{O} \mathcal{A}_{r}(E, F)$ it is a projection band.

\section{C-COMPACT AND NARROW ORTHOGONALLY ADDITIVE OPERATORS}

In this section we consider a new class of vector lattices, where the condition of Dedekind completeness is replaced by a much weaker property. For laterally-tonorm continuous, $C$-compact orthogonally additive operators from a $C$-complete vector lattice $E$ to a Banach space $X$ we show their narrowness.

Definition 4.1. A vector lattice $E$ is said to be $C$-complete, if for each $x \in E_{+}$ any subset $D \subset \mathcal{F}_{x}$ has a supremum and an infimum.

Clearly, every Dedekind complete vector lattice $E$ is $C$-complete. The reverse statement, in general, is not true.

5 This proposition can be applied since by Proposition 2.3 the vector lattice $\mathcal{O} \mathcal{A}_{r}(E, F)$ is Dedekind complete. Any $T$-step-function $S \in \mathcal{O} \mathcal{A}_{r}(E, F)$ has the form $S=\sum_{i=1}^{m} \lambda_{i} T_{i}$, where $T_{i}$ are disjoint fragments of $T$ such that $T=\bigsqcup_{i=1}^{m} T_{i}$.

6 This follows from the fact that together with $T$ each fragment $T_{i}$ of $T$ belongs to $\mathcal{C O} \mathcal{A}_{r}(E, F)$. 
Proposition 4.2. The vector lattice $E=C[0,1]$ of all continuous functions on the interval $[0,1]$ is $C$-complete.

Proof. Fix $f \in E_{+}$. Since the set $\mathcal{S}_{f}:=\{t \in[0,1]: f(t)>0\}$ is open, by [10, Chap.2, Theorem 5] there is the decomposition $\mathcal{S}_{f}=\bigcup_{i=1}^{\infty}\left(a_{i}, b_{i}\right)$ where $\left(a_{i}, b_{i}\right) \cap$ $\left(a_{j}, b_{j}\right)=\emptyset, i \neq j$. Take $g \in \mathcal{F}_{f}$. We claim that for every $i \in \mathbb{N}$ either $g(t)=0$ or $g(t)=f(t)$ for any $t \in\left(a_{i}, b_{i}\right)$. Indeed, denote by $f_{i}$ and $g_{i}$ the restrictions of $f$ and $g$ on the closed interval $\left[a_{i}, b_{i}\right]$, respectively. It is clear that $g_{i} \in \mathcal{F}_{f_{i}}$. Assume that there exists a nonzero fragment $v_{i} \in \mathcal{F}_{f_{i}}$ such that $v_{i} \perp g_{i}$ and $f_{i}=g_{i}+v_{i}$. Put $G_{i}:=\left\{t \in\left(a_{i}, b_{i}\right): g(t)>0\right\}$ and $V_{i}=\left\{t \in\left(a_{i}, b_{i}\right): v(t)>0\right\}$. Since $f_{i}$ is strictly positive on $\left(a_{i}, b_{i}\right)$ we deduce that $\left(a_{i}, b_{i}\right)=G_{i} \sqcup V_{i}$ and therefore $G_{i}$ and $V_{i}$ are open-closed subsets of $\left(a_{i}, b_{i}\right)$. But the interval $\left[a_{i}, b_{i}\right]$ is a connected set and we come to the contradiction. Thus $f_{i}$ has no fragment $0<v_{i}<f_{i}$ and therefore, either $f_{i}(t)=g_{i}(t)$ or $g_{i}(t)=0$ for any $t \in\left(a_{i}, b_{i}\right)$. With each $g \in \mathcal{F}_{f}$ there is associated the sequence $\left(g_{i}\right)_{i \in \mathbb{N}}$, where

$$
g_{i}=\left\{\begin{array}{ll}
1, & \text { if } g(t)=f(t) \\
0, & \text { if } g(t)=0
\end{array} \quad t \in\left(a_{i}, b_{i}\right) .\right.
$$

Clearly, in this way a one-to-one correspondence between $\mathcal{F}_{f}$ and the set of all 0 - 1 sequences is established. Let $D$ be any fixed subset of $\mathcal{F}_{f}$. Put

$$
\begin{gathered}
D_{+}:=\left\{i \in \mathbb{N}: \exists g \in D \text { such that } g_{i}=1\right\} ; \\
D_{-}:=\left\{i \in \mathbb{N}: \text { such that } g_{i}=1 \text { for } \forall g \in D\right\} .
\end{gathered}
$$

Consider the pair of sequences $\left(u_{i}\right)_{i \in \mathbb{N}}$ and $\left(v_{i}\right)_{i \in \mathbb{N}}$, where

$$
\begin{aligned}
& u_{i}= \begin{cases}1, & \text { if } i \in D_{+} \\
0, & \text { otherwise, }\end{cases} \\
& v_{i}= \begin{cases}1, & \text { if } i \in D_{-} \\
0, & \text { otherwise. }\end{cases}
\end{aligned}
$$

With $\left(u_{i}\right)_{i \in \mathbb{N}}$ and $\left(v_{i}\right)_{i \in \mathbb{N}}$ there is associated the pair $\{u, v\}$ of fragments of $f$. Clearly $u=\sup D$ and $v=\inf D$.

Since the vector lattice $C[0,1]$ is Archimedean but not Dedekind complete the previous proposition shows that the set of all $C$-complete vector lattices is a new subclass of vector lattices which strictly contains the class of all Dedekind complete vector lattices. We note that in general a $C$-complete vector lattice is not Archimedian. 
Example 4.3. Let $E=\mathbb{R}^{2}$ equipped with the lexicographic order. That is, we consider $E$ as a vector lattice with the following order ${ }^{7}\left(x_{1}, x_{2}\right) \geq\left(y_{1}, y_{2}\right)$, whenever either $x_{1}>y_{1}$ or else $x_{1}=y_{1}$ and $x_{2} \geq y_{2}$. The vector lattice $E$ is not Archimedian. On the other hand it is not hard to verify that the Boolean algebra of all fragments $\mathcal{F}_{x}$ of an arbitrary element $x=\left(x_{1}, x_{2}\right) \in E_{+}$contains only two elements:

$$
\mathcal{F}_{x}=\left\{\left(x_{1}, x_{2}\right),(0,0)\right\}
$$

Hence $E$ is $C$-complete.

We say that a set $D \subset E$ is laterally bounded, if there exits $x \in E$ such that $D \subset \mathcal{F}_{x}$. We say that a laterally bounded set $D$ has a lateral supremum (infimum) if there exists $u \in E(v \in E)$ such that $u=\sup D(v=\inf D)$ with respect to the partial order $\sqsubseteq$ in $\mathcal{F}_{x}$. Taking into account Proposition 3.10 we deduce that a vector lattice $E$ is $C$-complete if and only if every laterally bounded subset of $E$ has the lateral supremum and infimum.

Definition 4.4. Let $E$ be a vector lattice and $X$ be a normed space. An orthogonally additive operator $T: E \rightarrow X$ is called narrow, if for any $x \in E$ and $\varepsilon>0$ there exists a pair $x_{1}, x_{2}$ of mutually complemented fragments of $v$, such that $\left\|T x_{1}-T x_{2}\right\|<\varepsilon$. In particular, if $X=\mathbb{R}$, we call $T$ a narrow functional.

Observe that the image of an atom under a narrow operator $T$ is zero. Indeed. The only disjoint fragments of an atom $a$ are 0 and $a$. So, due to the narrowness of $T$, for any $\varepsilon>0$ one has $\|T u\|<\varepsilon$, what means $T u=0$.

This is the reason for supposing the vector lattice $E$ to be atomless in the Theorems 4.7 and in most of the propositions of the current section.

Example 4.5. Let $(\Omega, \Sigma, \mu)$ be a $\sigma$-finite measure space. Consider a map $\mathcal{N}$ : $L_{1}(\mu) \rightarrow \mathbb{R}$ defined by

$$
\mathcal{N}(f)=\|f\|_{L_{1}(\mu)}, \quad f \in L_{1}(\mu) .
$$

In [23] (Proposition 2.5) it was shown that $\mathcal{N}$ is a narrow orthogonally additive functional on $L_{1}(\mu)$.

A net $\left(x_{\alpha}\right)_{\alpha \in \Lambda}$ in a vector lattice $E$ laterally converges to $x \in E$ if $x_{\alpha} \sqsubseteq x_{\beta} \sqsubseteq x$ for all $\alpha \leq \beta$ and $x_{\alpha}$ order converges to $x$. This is written as $x_{\alpha} \stackrel{\text { lat }}{\longrightarrow} x$.

\footnotetext{
7 The cone $E_{+}$in this vector lattice consists of the open right half-space $\left\{\left(x_{1}, x_{2}\right): x_{1}>0\right\}$ joint with the half-ray $\left\{\left(x_{1}, x_{2}\right): x_{1}=0, x_{2} \geq 0\right\}$.
} 
Definition 4.6. An orthogonally additive operator $T$ from a vector lattice $E$ to a normed space $X$ is called laterally-to-norm continuous whenever for each laterally convergent net $\left(x_{\alpha}\right)_{\alpha \in \Lambda}$ with $x_{\alpha} \stackrel{\text { lat }}{\longrightarrow} x$ the net $\left(T x_{\alpha}\right)_{\alpha \in \Lambda}$ converges with respect to the norm to in $X$ to $T x$.

The following theorem is the second main result of the article.

Theorem 4.7. Let $E$ be an atomless $C$-complete vector lattice and $X$ be a Banach space. Then every orthogonally additive laterally-to-norm continuous $C$-compact operator $T: E \rightarrow X$ is narrow.

The next auxiliary proposition is well known (see e.g. [21, Lemma 10.20]).

Proposition 4.8. Let $\left(v_{i}\right)_{i=1}^{n}$ be a finite subset of elements in a finite dimensional normed space $V$ and $\left(\lambda_{i}\right)_{i=1}^{n}$ be non-negative numbers such that $0 \leq \lambda_{i} \leq 1$ for each $i$. Then there exists a set $\left(\theta_{i}\right)_{i=1}^{n}$ of numbers such that $\theta_{i} \in\{0,1\}, i \in\{1, \ldots, n\}$ and

$$
\left\|\sum_{i=1}^{n}\left(\lambda_{i}-\theta_{i}\right) v_{i}\right\| \leq \frac{\operatorname{dim} V}{2} \max _{i \in\{1, \ldots, n\}}\left\|v_{i}\right\| .
$$

Proposition 4.9. Let be $E$ an atomless vector lattice, $x \in E, X$ a Banach space and $T: E \rightarrow X$ an orthogonally additive laterally-to-norm continuous operator. Then for any $\varepsilon>0$ there exists a decomposition $x=y \sqcup z$, where $y, z$ are nonzero fragments of $x$ such that $\|T z\|<\varepsilon$.

Proof. Since $E$ is an atomless vector lattice the Boolean algebra $\mathfrak{A}:=\mathcal{F}_{x}$ has infinite cardinality. We note that the set of all fragments of $x$ is the net $\left(x_{\alpha}\right)_{\alpha \in \mathfrak{A}}$ laterally converges to $x$. The laterally-to-norm continuity of $T$ implies the existence of $\alpha_{0} \in \mathfrak{A}$ such that $\left\|T x-T x_{\alpha}\right\|<\varepsilon$ for all $\alpha \geq \alpha_{0}$. Consider for $\alpha_{0}$ the disjoint decomposition $x=\left(x-x_{\alpha_{0}}\right) \sqcup x_{\alpha_{0}}$. Then

$$
T x=T\left(\left(x-x_{\alpha_{0}}\right) \sqcup x_{\alpha_{0}}\right)=T\left(x-x_{\alpha_{0}}\right)+T x_{\alpha} \quad \text { implies } \quad\left\|T\left(x-x_{\alpha_{0}}\right)\right\|<\varepsilon .
$$

Assign $y:=x_{\alpha_{0}}$ and $z=x-x_{\alpha_{0}}$. Then $\|T z\|<\varepsilon$ and $x=y \sqcup z$ is the desirable disjoint decomposition.

Proposition 4.10. Let be $E$ an atomless vector lattice, $x \in E, X$ a Banach space and $T: E \rightarrow X$ an orthogonally additive laterally-to-norm continuous operator. Assume that $\left(y_{n}\right)_{n \in \mathbb{N}}$ is a sequence of fragments of $x$ such that $y_{1}=x, y_{n} \sqsubseteq y_{m}$ for $n \geq m ; m, n \in \mathbb{N}$ and $\bigcap_{n \in \mathbb{N}} \mathcal{F}_{y_{n}}=\{0\}$. Then $\lim _{n \rightarrow \infty}\left\|T y_{n}\right\|=0$. 
Proof. By definition $y_{m}=y_{n} \sqcup\left(y_{m}-y_{n}\right)$ for $m, n \in \mathbb{N}$ with $n \geq m$. For $x_{n}:=$ $x-y_{n}, n \in \mathbb{N}$ with $y_{n}=y_{m}-\left(y_{m}-y_{n}\right)$ we can write

$$
x_{n}=x-y_{m}+\left(y_{m}-y_{n}\right)=x-y_{m}+\left(\left(x-y_{n}\right)-\left(x-y_{m}\right)\right)=x_{m}+\left(x_{n}-x_{m}\right) .
$$

Clearly $x_{m} \perp\left(x_{n}-x_{m}\right)$ for $n, m \in \mathbb{N}$ and $n \geq m$ and therefore $x_{n} \perp\left(x-x_{n}\right)$ for all $n \in \mathbb{N}$. Thus $x=x_{n} \sqcup\left(x-x_{n}\right), n \in \mathbb{N}$ and $\bigcap_{n \in \mathbb{N}} \mathcal{F}_{x-x_{n}}=\bigcap_{n \in \mathbb{N}} \mathcal{F}_{y_{n}}=\{0\}$. Hence we deduce that the sequence $\left(x_{n}\right)_{n \in \mathbb{N}}$ laterally converges $^{8}$ to $x$. Now the relation $x_{n} \perp\left(x-x_{n}\right)$ for all $n \in \mathbb{N}$ implies

$$
T x=T\left(x_{n} \sqcup\left(x-x_{n}\right)\right)=T x_{n}+T\left(x-x_{n}\right) \text { and } T\left(x-x_{n}\right)=T x-T x_{n} .
$$

Thus by laterally-to-norm continuity of $T$ we have that $T x_{n}$ is norm convergent to $T x$ in $\mathrm{X}$ and

$$
\lim _{n \rightarrow \infty}\left\|T x-T x_{n}\right\|=\lim _{n \rightarrow \infty}\left\|T y_{n}\right\|=0
$$

Proposition 4.11. Let be $E$ an atomless $C$-complete vector lattice, $X$ a Banach space, $T: E \rightarrow X$ an orthogonally additive laterally-to-norm continuous operator, $x \in E$ and $\varepsilon>0$. Then for some $n \in \mathbb{N}$ there exists a decomposition $x=\bigsqcup_{i=1}^{n} x_{i}$, where $x_{i}$ are nonzero fragments of $x$ such that $\left\|T x_{i}\right\| \leq \varepsilon$ for any $i \in\{1, \ldots, n\}$.

Proof. By Proposition 4.9 the set

$$
D_{x, T, \varepsilon}:=\left\{z \in \mathcal{F}_{x}: z \neq 0,\|T z\| \leq \varepsilon\right\}
$$

is not empty. We note that $D_{x, T, \varepsilon}$ is a partially ordered set with respect to the relation $\sqsubseteq$. Let $\left(u_{\lambda}\right)_{\lambda \in \Lambda} \subseteq D_{x, T, \varepsilon}$ be a chain, where $\Lambda$ is a some linearly ordered index set. Clearly $u_{\mu} \sqsubseteq u_{\lambda}$ for all $\mu, \lambda \in \Lambda, \mu \sqsubseteq \lambda$. By the $C$-completeness of vector lattice $E$ there exists $u=(o)-\lim _{\lambda \in \Lambda}\left(u_{\lambda}\right)$, where $u \in \mathcal{F}_{x}$. Due to $\left\|T u_{\lambda}\right\| \leq \varepsilon$ and the laterally-to-norm continuity of $T$ we have that then $T u=\lim _{\lambda \in \Lambda}\left(T u_{\lambda}\right)$ and therefore $u \in D_{x, T, \varepsilon}$. By Zorn's Lemma, there is a maximal element ${ }^{9} z \in$ $D_{x, T, \varepsilon}$ with $\|T z\| \leq \varepsilon$. Put $y=x-z$. If $\|T y\| \leq \varepsilon$ then we got the required decomposition of $x$. Otherwise we apply Proposition 4.9 to $y$ and get $y=y_{1} \sqcup y_{2}$, where $y_{1}$ is a maximal element in $D_{y, T, \varepsilon}$ with $\left\|T y_{1}\right\| \leq \varepsilon$. In case of necessity, i.e. if $\left\|T y_{2}\right\|>\varepsilon$, by further continuing in the same way with the corresponding fragments $y_{2}, y_{4}, \ldots, y_{2 k}, \ldots$ of $y$ we construct a sequence of decompositions $y_{2 k}=$ $y_{2 k+1} \sqcup y_{2 k+2}$, where $y_{2 k+1}$ is a maximal element in $D_{y_{2 k}, T, \varepsilon}$ and satisfying the

\footnotetext{
8 Indeed, if some vector $0 \neq u$ is a fragment of each vector $x-x_{n}$ then $u \sqsubseteq\left(x-x_{n}\right)$ and $\left|x-x_{n}\right| \leq u_{n}$ for some sequence $u_{n} \downarrow 0$ is impossible.

9 The element $z$ is maximal in $D_{x, T, \varepsilon}$, if $\nexists u \in D_{x, T, \varepsilon}$ such that $z \sqsubseteq u$ and $z \neq u$.
} 
conditions $\left\|T y_{2 k+1}\right\| \leq \varepsilon$ and $\left\|T y_{2 k+2}\right\|>\varepsilon, k \in \mathbb{N}$. We claim that there exists $l \in \mathbb{N}$ such that $y_{2 l}=y_{2 l+1} \sqcup y_{2 l+2}$ and both $\left\|T y_{2 l+1}\right\|,\left\|T y_{2 l+2}\right\| \leq \varepsilon$. Assume the contrary. Then the sequence $\left(y_{2 k}\right)_{k \in \mathbb{N}}$ of fragments of $y$ is such that $\left\|T y_{2 k}\right\|>\varepsilon$ for all $k \in \mathbb{N}$. Nevertheless we show $\bigcap_{k \in \mathbb{N}} \mathcal{F}_{y_{2 k}}=\{0\}$. Indeed, assume that there exists a nonzero element $v \in \bigcap_{k \in \mathbb{N}} \mathcal{F}_{y_{2 k}}$. Then for the sequence

$$
y_{2}^{\prime}=y_{2}-v, y_{4}^{\prime}=y_{4}-v, \ldots, y_{2 k}^{\prime}=y_{2 k}-v, \ldots
$$

we have $y_{2 n}^{\prime} \sqsubseteq y_{2 m}^{\prime}$ for $m, n \in \mathbb{N}$ with $n \geq m$ and $\bigcap_{n \in \mathbb{N}} \mathcal{F}_{y_{2 n}^{\prime}}=\{0\}$. According to Proposition 4.10 there exists $n_{0} \in \mathbb{N}$ such that $\left\|T y_{2 n_{0}}^{\prime}\right\|<\varepsilon$. Thus $y_{2 n_{0}}=y_{2 n_{0}}^{\prime} \sqcup v$, $y_{2 n_{0}}^{\prime} \in D_{y_{2 n_{0}}, T, \varepsilon}$ and $y_{2 n_{0}+1}$ is a maximal element of $D_{y_{2 n_{0}}, T, \varepsilon}$. We have

$$
y_{2 n_{0}}=y_{2 n_{0}+1} \sqcup y_{2 n_{0}+2}=y_{2 n_{0}}^{\prime} \sqcup v \text {. }
$$

Consider now the two cases:

$$
\mathcal{F}_{y_{2 n_{0}+1}} \cap \mathcal{F}_{v}=\{0\} \quad \text { and } \quad \mathcal{F}_{y_{2 n_{0}+1}} \cap \mathcal{F}_{v} \neq\{0\}
$$

In the first case, since no non-zero fragment of $v$ is a fragment of $y_{2 n_{0}+1}$, we get $y_{2 n_{0}+1} \sqsubseteq y_{2 n_{0}}^{\prime}$. Observe that - due to the maximality of $y_{2 n_{0}+1}$ in $D_{y_{2 n_{0}}, T, \varepsilon}$ - the relation $y_{2 n_{0}+1} \sqsubseteq y_{2 n_{0}}^{\prime}, y_{2 n_{0}+1} \neq y_{2 n_{0}}^{\prime}$ is impossible. Hence $y_{2 n_{0}}^{\prime}=y_{2 n_{0}+1}$, i.e. $y_{2 n_{0}+2}=v$ and, therefore $v$ is not a fragment of $y_{2 n_{0}+4}$. In the second one there is a nonzero fragment $w \in \mathcal{F}_{y_{2 n_{0}+1}} \cap \mathcal{F}_{v}$. Thus $v=(v-w) \sqcup w$ and $w \sqsubseteq y_{2 n_{0}+1}$, i.e. the non-zero fragment $w$ of $v$ belongs to $\mathcal{F}_{y_{2 n_{0}+1}}$. Therefore the element $v$ can not be a fragment of $y_{2 n_{0}+2}$.

Hence $\bigcap_{k \in \mathbb{N}} \mathcal{F}_{y_{2 k}}=\{0\}$ and so, again by applying Proposition 4.10, we get $\lim _{k \rightarrow \infty}\left\|T y_{2 k}\right\|=0$. This is a contradiction and therefore the desirable $l \in \mathbb{N}$ exists. Consider the following elements

$$
x_{1}=y_{1}, x_{2}=y_{3}, \ldots, x_{l}=y_{2 l-1}, x_{l+1}=y_{2 l+1}, x_{l+2}=y_{2 l+2}, x_{l+3}=z \text {. }
$$

Then $x=\bigsqcup_{i=1}^{n} x_{i}$ with $n=l+3$ is the desirable decomposition of $x$.

Proposition 4.12. Let $E$ be an atomless $C$-complete vector lattice and $V$ a finite dimensional Banach space. Then every orthogonally additive laterally-to-norm continuous operator $G: E \rightarrow V$ is narrow.

Proof. Fix any $x \in E$ and $\varepsilon>0$. According to Proposition 4.11 there is a disjoint decomposition $x=\bigsqcup_{i=1}^{n} x_{i}$ such that $\left\|G x_{i}\right\|<\frac{\varepsilon}{\operatorname{dim} V}$ for any $i \in\{1, \ldots, n\}$. Then by 
using Proposition 4.8 for $\lambda_{i}=\frac{1}{2}$ there exist numbers $\theta_{i} \in\{0,1\}$ for $i \in\{1, \ldots, n\}$ such that

$$
2\left\|\sum_{i=1}^{n}\left(\frac{1}{2}-\theta_{i}\right) G x_{i}\right\| \leq \operatorname{dim} V \max _{i \in\{1, \ldots, n\}}\left\|G x_{i}\right\|<\varepsilon .
$$

Observe that for $I_{0}=\left\{i \in\{1, \ldots, n\}: \theta_{i}=0\right\}$ and $I_{1}=\left\{i \in\{1, \ldots, n\}: \theta_{i}=1\right\}$ the vectors $y_{k}=\bigsqcup_{i \in I_{k}} x_{i}$ for $k \in\{0,1\}$ are mutually complemented fragments of $x$ and by (4.1),

$$
\left\|G y_{1}-G y_{2}\right\|=\left\|\sum_{i \in I_{0} \sqcup I_{1}}\left(1-2 \theta_{i}\right) G x_{i}\right\|<\varepsilon,
$$

hence the operator $G$ is narrow.

Definition 4.13. Let $E$ be a vector lattice and $F$ a vector space. An orthogonally additive operator $T: E \rightarrow F$ is called of finite rank if the set $T(E)$ generates a finite-dimensional subspace in $F$.

Now we are in the position to prove the second main result (Theorem 4.7). Before, however, we notice that a Banach space $X$ can be considered as a closed subspace of the Banach space

$$
W:=l_{\infty}\left(B_{X^{*}}\right)=\left\{q: B_{X^{*}} \rightarrow \mathbb{R}, \sup |q(f)|<\infty, f \in B_{X^{*}}\right\}
$$

(equipped with the supremum-norm) of all real-valued bounded functions on the closed unit ball $B_{X^{*}}$ of the dual space $X^{*}$ according to

$$
X \hookrightarrow X^{* *} \hookrightarrow W
$$

where the notation $\hookrightarrow$ means the isometric embedding

$$
X \ni x \mapsto F_{x} \in X^{* *} \quad \text { given by } \quad F_{x}(f):=f(x), f \in B_{X^{*}} .
$$

Observe that, if $H$ is a relatively compact subset of the Banach space $W$ and $\varepsilon>0$, then there exists a linear finite rank operator $R \in \mathcal{L}(W) \operatorname{such}^{10}$ that $\|w-R w\| \leq \varepsilon$ for every $w \in H[21$, Lemma 10.25].

Proof of Theorem 4.7. Fix an arbitrary $x \in E$ and $\varepsilon>0$. Due to the $C$ compactness of $T$ the set $K=T\left(\mathcal{F}_{x}\right)$ is relatively compact in $X$ and therefore in $W$. It follows that there exists a finite rank operator $S \in \mathcal{L}(W)$ such that $\|y-S y\| \leq \frac{\varepsilon}{4}$ for every $y \in K$. Then $G=S \circ T$ is an orthogonally additive laterally-to-norm continuous finite rank operator. By Lemma $4.12 G$ is narrow

\footnotetext{
10 By $\mathcal{L}(W)$ there is denoted the space of all linear bounded operators on $W$.
} 
and consequently, there exist mutually complemented fragments $x_{1}, x_{2}$ of $x$ such that $\left\|G x_{1}-G x_{2}\right\|<\frac{\varepsilon}{2}$. Therefore,

$$
\begin{aligned}
& \left\|T x_{1}-T x_{2}\right\|= \\
& \left\|T x_{1}-T x_{2}+S\left(T x_{1}\right)-S\left(T x_{2}\right)-S\left(T x_{1}\right)+S\left(T x_{2}\right)\right\|= \\
& \left\|T x_{1}-T x_{2}+G x_{1}-G x_{2}-G x_{1}+G x_{2}\right\| \leq \\
& \left\|G x_{1}-G x_{2}\right\|+\left\|T x_{1}-G x_{1}\right\|+\left\|T x_{2}-G x_{2}\right\|<\frac{\varepsilon}{2}+\frac{\varepsilon}{2}=\varepsilon
\end{aligned}
$$

since $\left\|T x_{i}-G x_{i}\right\|<\frac{\varepsilon}{4}$ for $i \in\{1,2\}$. This completes the proof.

We remark that Theorem 4.7 generalizes the result of the article [18, Theorem 3.2].

Acknowledgments. Marat Pliev was supported by the Russian Foundation for Basic Research (grant number 17-51-12064). Martin Weber was supported by the Deutsche Forschungsgemeinschaft (grant number CH 1285/5-1, Order preserving operators in problems of optimal control and in the theory of partial differential equations).

\section{REFERENCES}

[1] N. Abasov, M. Pliev, On extensions of some nonlinear maps in vector lattices, J. Math. Anal. Appl. 455 (2017), 516-527.

[2] N. Abasov, M. Pliev, Disjointness preserving orthogonally additive operators in vector lattices, Banach of Math. Anal. 12 (2018), 3, 730-750.

[3] N. Abasov, M. Pliev, Dominated orthogonally additive operators in lattice-normed spaces, Advances in Operator Theory, 4 (2019), 1, 251-264.

[4] C. D. Aliprantis, O. Burkinshaw, Positive Operators, Springer, Dordrecht. (2006).

[5] J. A. Appell, P. P. Zabrejko, Nonlinear superposition operators, Cambridge University Press, 2008.

[6] W. A. Feldman, Lattice preserving maps on lattices of continuous functions, J. Math. Anal. Appl., 404 (2013), 310-316.

[7] W.A. Feldman, A characterization of non-linear maps satisfying orthogonality properties, Positivity 21 (2017), 1, 85-97.

[8] W. A. Feldman, A factorization for orthogonally additive operators on Banach lattices, J. Math. Anal. and Appl., 472, (2019), 1, 238-245.

[9] O. Fotiy, A. Gumenchuk, I. Krasikova, M. Popov, On sums of narrow and compact operators, Positivity, doi.org/10.1007/s11117-019-00666-4.

[10] A. N. Kolmogorov, S. V. Fomin, Introductory Real Analysis, Dover Publication. (1975).

[11] O. Maslyuchenko, V. Mykhaylyuk, M. Popov, A lattice approach to narrow operators, Positivity, 13 (2009), 459-495.

[12] J. M. Mazón, S. Segura de León, Order bounded ortogonally additive operators, Rev. Roumane Math. Pures Appl., 35 (1990), 4, 329-353. 
[13] J. M. Mazón, S. Segura de León, Uryson operators, Rev. Roumane Math. Pures Appl. 35 (1990), 5, 431-449.

[14] V. Mykhaylyuk, M. Popov, On sums of narrow operators on Köthe function space, J. Math. Anal. Appl. 404 (2013), 554-561.

[15] V. Mykhaylyuk, On the sum of narrow and a compact operators, J. Funct. Anal. 266 (2014), 5912-5920.

[16] V. Orlov, M. Pliev, D. Rode, Domination problem for AM-compact abstract Uryson operators, Archiv der Mathematik, 107, 5, (2016), 543-552.

[17] Plichko A., M.Popov M. Symmetric function spaces on atomless probability spaces, Dissertationes Math. (Rozprawy Mat.), 306 (1990), 1-85.

[18] M. Pliev, M. Popov, Narrow orthogonally additive operators, Positivity, 18 (2014), 4, 641667.

[19] M. Pliev, K. Ramdane Order unbounded orthogonally additive operators in vector lattices, Mediterranean J. Math. 15 (2018), 2, 20 p.

[20] M. Pliev, X. Fang, Narrow orthogonally additive operators in lattice-normed spaces, Siberian Math. J., 58 (2017), 1, 134-141.

[21] M. Popov, B. Randrianantoanina, Narrow Operators on Function Spaces and Vector Lattices, De Gruyter Studies in Mathematics 45, De Gruyter (2013).

[22] M. A. Pliev, M. R. Weber, Disjointness and order projections in the vector lattices of abstract Uryson operators, Positivity, 20 (2016), 3, 695-707.

[23] M. A. Pliev, F. Polat, M. R. Weber, Narrow and C-compact orthogonally additive operators in lattice-normed spaces, Results in Math., 74 (2019), 4, 19 pp.

[24] Tradacete, P., and Villanueva, I. Continuity and representation of valuations on star bodies, Adv. Math. 329 (2018), 361-391.

[25] Tradacete, P., and Villanueva, I. Valuations on Banach lattices, Int. Math. Res. Not., (2019), to appear.

[26] A. C. Zaanen, Introduction to Operator Theory in Riesz Spaces, Springer, Berlin Heidelberg New York. (1997).

Southern Mathematical Institute of the Russian Academy of Sciences, Vladikavkaz, 362027 Russia

E-mail address: maratpliev@gmail.com

Technical University Dresden, Department of Mathematics, Institute of Analysis, 01062 Dresden, Germany

E-mail address: martin.weber@tu-dresden.de 